\title{
Evaluación del mercurio en jurel proveniente de Matarani, Vila Vila e Ilo en Arequipa.
}

\section{Evaluation of the Mercury in coming jurel of Matarani, Vila Vila And Ilo In Arequipa}

\author{
Anyelina $\mathrm{Paz}^{1}$
}

http://dx.doi.org/10.21503/CienciayDesarrollo.2013.v16i1.04

\section{RESUMEN}

El trabajo de investigación tuvo como objetivo determinar la presencia de metil mercurio en especies de alto consumo popular, así como las zonas de presencia, eligiéndose Matarani, Vila Vila e Ilo, por ser los lugares de mayor abastecimiento del recurso marino para la población de Arequipa. También se evaluó la época para obtener una relación directa con las estaciones de mayor precipitación y la remoción del metal a las zonas costeras.

Las muestras de jurel se acopiaron en el centro de abastos de la ciudad proveniente de las tres localidades, estibaron y refrigeraron para conservar su calidad y permitir el análisis. Se empleó para la determinación de la concentración de metil mercurio el espectrofotómetro de absorción atómica; repitiéndose los análisis para las otras estaciones del año.

Los resultados obtenidos se analizaron empleando el análisis de varianza, el Test de amplitud de Duncan y la T-Student; para identificar si los resultados obtenidos de cada localidad y por estación son significativos y además de identificar la zona que provee de los peces más contaminados con metil mercurio

Palabras clave: jurel, metil mercurio, bioacumulación, contaminación, espectrofotómetro de absorción atómica.

\section{ABSTRACT}

This research work was aimed to determine the presence of methyl mercury in popular guzzling species and areas of presence, whichever Matarani, Vila Vila and Ilo, being the places of marine resource supply for the population of Arequipa. We also evaluated the time to get a direct link with the highest rainfall seasons and metal removal to coastal areas.

Horse mackerel samples were collected in the center of the city supplies from the three localities, loaded and refrigerated to preserve its quality and allow analysis. It was employed for determining the concentration of methyl mercury, atomic absorption spectrophotometer, repeating the analysis for the other seasons.

Results were analyzed employing analysis of variance, test of Duncan and T-Student to identify if these results of each locality and for each seasons are important and identify area that provide fish more pollution with methyl mercury.

Keywords: Horse mackerel, methyl mercury, bioaccumulation, contamination, atomic absorption spectrophotometer. 


\section{INTRODUCCIÓN}

Dada la gran cantidad de insumos empleados en la actualidad en diversos procesos productivos y el mal manejo de los mismos, al emplear técnicas deficientes tales como en la minería artesanal del oro la cual emplea procedimientos rudimentarios para su extracción.

En el proceso de amalgamación, el oro es atrapado por el mercurio en el seno de una pulpa acuosa para formar una sustancia altamente viscosa y de color blanco brillante, denominada amalgama. La recuperación final del metal precioso se realiza mediante un fuerte calentamiento de la aleación (evaporación del mercurio) o la utilización de ácido nítrico (disolución del mercurio); generando la contaminación de los agentes ambientales, en este caso el agua.

El jurel es una especie de alto consumo por el público arequipeño. Se debe indicar que en las zonas altas de la ciudad, la extracción artesanal del oro es una actividad económica importante, por lo que existe la posibilidad de encontrar metil mercurio en los peces que se expenden en los mercados locales. Para la evaluación se tomaron 15 muestras de la especie (hígado y cerebro) por cada estación del año, en las cuales se evaluaron su concentración de mercurio, utilizando la técnica del espectrofotómetro de absorción atómica

\section{MATERIALES Y MÉTODOS.}

\section{Material}

- Celda espectrofotométrica de cuarzo de flujo continuo

- Equipo de aereación. Tubo de aereación

- Trampa de agua

- Llave de tres pasos o tres vías

- Medidor de flujo con un rango de 0-1.7 1/min

- Platina de calentamiento con agitador magnético

- Baño de vapor. Licuadora. Autoclave
- Espectrofotómetro de absorción atómica, equipado con corrector de fondo.

- Generador de hidruros para absorción atómica

- Balanza granataria con sensibilidad de $0.01 \mathrm{~g}$

\section{Método}

Las pruebas fueron realizadas en el laboratorio A\&E PHARMA S.R.L. de Arequipa y corresponden a las estaciones de verano, otoño, invierno y primavera del año 2005 (Considerándolo en la actualidad como un trabajo referencial por el tiempo transcurrido). Se tomaron quince muestras por estación, las que se estibaron en hielo y se enviaron al laboratorio a analizar el hígado y el cerebro. Los resultados de las pruebas se analizaron con la varianza y la $\mathrm{T}$ student.

Se extrajo el hígado y cerebro de cada especie. Todos los reactivos químicos fueron de carácter analítico. Para la digestión se emplean soluciones de Ácido Nítrico (1,5\%v/v) y de bromohídruro de sodio ( $3 \% \mathrm{v} / \mathrm{v})$, ácido erclórico, ácido sulfúrico y peróxido de hidrógeno. La solución base de mercurio aproximadamente $(1000 \mathrm{mg} / \mathrm{l})$ se prepara a partir de óxido de mercurio (II).

Los tejidos de hígado y cerebro se secan en un liofilizador a $-5^{\circ} \mathrm{C}$ por seis horas. Se empleó un Espectrómetro de absorción atómica con vapor frío equipado con una lámpara catódica de mercurio, que opera a $6 \mathrm{~mA}$ (con un ancho espectral de $0,7 \mathrm{~nm}$ ) y una longitud de resonancia de 253,6 nm. Los valores se expresan en $\mu \mathrm{g} \mathrm{Hg} \mathrm{g}^{-1} \mathrm{de}$ tejido (peso seco).

Pesar de 0,5 a $0,6 \mathrm{~g}$ de muestra en un matraz erlenmeyer de $125 \mathrm{ml}$. de igual forma pesar tejido blanco y muestra para fortificar. Preparar ocho matraces Erlenmeyer de $125 \mathrm{ml}$. para los estándares de calibración. Adicionar a todos los matraces $10 \mathrm{ml}$ de $\mathrm{HNO} 3$ al25\%, tapar y sellar. Colocar en autoclave a $125^{\circ} \mathrm{C}$ o 15 libras por 30 min. Enfriar y analizar por absorción atómica. 


\section{RESULTADOS}

Tabla 1. Muestreo en la estación de primavera

\begin{tabular}{cccc} 
ANÁLISIS & Matarani $(\mathrm{mg} / \mathrm{Kg})$ & Ilo $(\mathrm{mg} / \mathrm{Kg})$ & Vila Vila $(\mathrm{mg} / \mathrm{Kg})$ \\
\hline 1 & 7 & 7,4 & 6,9 \\
2 & 7,2 & 7,9 & 6,5 \\
3 & 7,8 & 8,5 & 6,2 \\
4 & 8,5 & 8,4 & 7 \\
\hline 5 & 8,4 & 8,9 & 6,9 \\
6 & 8,9 & 8,7 & 7 \\
\hline 7 & 9 & 7,2 & 6,9 \\
8 & 7,6 & 7,3 & 6,6 \\
9 & 7,4 & 7,5 & 6,9 \\
\hline 10 & 7,9 & 7,7 & 5,9 \\
\hline 11 & 8,4 & 9 & 6,5 \\
\hline 12 & 8,9 & 8,4 & 6,3 \\
\hline 13 & 8,1 & 8,6 & 6,8 \\
14 & 8,2 & 8,8 & 6,2 \\
\hline 15 & 9 & 7 & 7 \\
\hline
\end{tabular}

Fuente: Elaboración propia

\section{Tabla 2. Muestreo en la estación de verano}

\begin{tabular}{cccc} 
ANÁLISIS & Matarani $(\mathrm{mg} / \mathrm{Kg})$ & Ilo $(\mathrm{mg} / \mathrm{Kg})$ & Vila Vila $(\mathrm{mg} / \mathrm{Kg})$ \\
\hline 1 & 8,80 & 8,70 & 6,90 \\
2 & 8,40 & 8,30 & 7,00 \\
3 & 9,00 & 9,50 & 8,00 \\
4 & 8,10 & 8,00 & 7,20 \\
5 & 7,90 & 8,50 & 7,50 \\
6 & 8,70 & 8,20 & 7,10 \\
7 & 9,60 & 9,30 & 7,00 \\
8 & 9,90 & 9,20 & 7,90 \\
9 & 10,00 & 9,10 & 6,80 \\
10 & 9,10 & 9,00 & 6,70 \\
11 & 9,50 & 9,20 & 8,10 \\
12 & 9,00 & 9,20 & 8,20 \\
13 & 9,50 & 8,00 & 6,90 \\
14 & 8,10 & 7,90 & 7,20 \\
15 & 8,00 & 7,90 & 7,30 \\
\hline
\end{tabular}

Fuente: Elaboración propia 
Tabla 3. Muestreo en la estación de otoño

\begin{tabular}{cccc} 
ANÁLISIS & Matarani $(\mathrm{mg} / \mathrm{Kg})$ & Ilo $(\mathrm{mg} / \mathrm{Kg})$ & Vila Vila $(\mathrm{mg} / \mathrm{Kg})$ \\
\hline 1 & 7,50 & 7,40 & 6,50 \\
2 & 9,50 & 7,90 & 6,80 \\
3 & 7,90 & 8,00 & 7,50 \\
4 & 9,20 & 8,90 & 7,00 \\
\hline 5 & 9,40 & 9,50 & 7,20 \\
6 & 7,60 & 9,20 & 6,90 \\
7 & 7,90 & 8,60 & 6,70 \\
8 & 8,00 & 7,90 & 6,40 \\
9 & 8,50 & 7,50 & 7,40 \\
10 & 8,90 & 7,60 & 7,30 \\
11 & 8,80 & 9,00 & 7,10 \\
12 & 7,60 & 9,10 & 6,50 \\
13 & 9,50 & 8,40 & 6,90 \\
14 & 9,50 & 8,20 & 6,80 \\
\hline 15 & 9,10 & 8,10 & 6,90 \\
\hline
\end{tabular}

Fuente: Elaboración propia

Tabla 4. Muestreo en la estación de invierno

\begin{tabular}{cccc} 
ANÁLISIS & Matarani $(\mathrm{mg} / \mathrm{Kg})$ & Ilo $(\mathrm{mg} / \mathrm{Kg})$ & Vila Vila $(\mathrm{mg} / \mathrm{Kg})$ \\
\hline 1 & 6,10 & 6,00 & 5,00 \\
\hline 2 & 6,50 & 6,50 & 5,20 \\
3 & 7,50 & 6,30 & 5,90 \\
4 & 7,90 & 6,90 & 6,00 \\
\hline 5 & 8,00 & 8,00 & 6,50 \\
6 & 6,10 & 7,50 & 6,30 \\
\hline 7 & 6,50 & 7,60 & 6,40 \\
\hline 8 & 6,90 & 7,40 & 5,00 \\
9 & 7,50 & 7,00 & 5,50 \\
10 & 7,10 & 6,90 & 5,90 \\
\hline 11 & 8,00 & 6,80 & 6,20 \\
12 & 7,90 & 6,70 & 5,00 \\
13 & 7,40 & 7,80 & 5,90 \\
14 & 6,40 & 7,50 & 5,50 \\
\hline 15 & 6,50 & 6,80 & 5,80 \\
\hline
\end{tabular}

Fuente: Elaboración propia 


\section{ANÁLISIS Y DISCUSIÓN}

Los resultados analizados determinaron la significancia entre los resultados obtenidos por localidad y por estación.

Tabla 5. Análisis de varianza para la primavera

\begin{tabular}{|c|c|c|c|c|c|c|}
\hline \multirow{2}{*}{$\begin{array}{l}\text { Fuente de } \\
\text { variación }\end{array}$} & \multirow[b]{2}{*}{ GI } & \multirow[b]{2}{*}{ SC } & \multirow[b]{2}{*}{$\mathrm{CM}$} & \multicolumn{3}{|c|}{ Cálculo de F } \\
\hline & & & & F calculado & F tabulado & F tabulado \\
\hline & & & & & $5 \%$ & $1 \%$ \\
\hline Lugar & 2 & 21,94 & 10,9687 & 35,02 & 3,33 & 5,42 \\
\hline Análisis & 14 & 5,70 & 0,4072 & 1,30 & 2,05 & 2,75 \\
\hline Error & 28 & 8,77 & 0,313190476 & & & \\
\hline Total & 44 & 36,41 & 0,8275 & & & \\
\hline
\end{tabular}

Fuente: Elaboración propia

En cuanto al lugar hay significancia, mientras que el análisis de los datos por localidad no tienen significancia.

\section{Test de amplitud múltiple Duncan}

\section{DUNCAN}

Sx $=$ Desviación Standar

$$
\begin{aligned}
& S_{x}=\sqrt{\frac{\text { CM error }}{\text { panelista }}} \\
& S_{x}=\sqrt{\frac{0,001764}{15}}=0,144497
\end{aligned}
$$

Promedios

2 Posiciones 3 Posiciones

$\begin{array}{ll}\text { S1 } & 8,1533 \\ \text { S2 } & 8,0867 \\ \text { S3 } & 6,6400\end{array}$

I a II 2 posiciones

I a III 3 posiciones

II a III 2 posiciones

\begin{tabular}{|c|c|c|c|c|c|}
\hline I a II & 2 & $-0,07$ & $<$ & 0,42 & NOSIG \\
\hline I a III & 3 & $-1,51$ & $<$ & 0,44 & NOSIG \\
\hline II a III & 2 & 1,45 & $>$ & 0,42 & SIG \\
\hline
\end{tabular}

\begin{tabular}{l|ccc}
\multirow{2}{*}{$\mathrm{RX}$} & $5 \%$ & 2,9 & 3,04 \\
& $1 \%$ & 3,91 & 4,08 \\
\multirow{3}{*}{$\mathrm{SxRx}$} & $5 \%$ & 0,42 & 0,44 \\
& $1 \%$ & 0,56 & 0,59 \\
\hline
\end{tabular}


Finalmente al no notar una amplitud y al dar el de Matarani con 8,15mg/Kg (mg de mercurio como amplitud los tres lugares se procede a por Kg de muestra) escoger el valor con mayor promedio que sería

Tabla 6. Análisis de varianza para el verano

ANVA

\begin{tabular}{ccccccc}
$\begin{array}{c}\text { Fuente de } \\
\text { variación }\end{array}$ & GI & SC & CM & F calculado & F tabulado & F tabulado \\
\hline Lugar & 2 & 21,94 & 10,9716 & 50,44 & 3,33 & 5,42 \\
\hline Análisis & 14 & 8,98 & 0,6412 & 2,95 & 2,05 & 2,75 \\
\hline Error & 28 & 6,09 & 0,217507937 & & & \\
\hline Total & 44 & 37,01 & 0,8411 & & & \\
\hline
\end{tabular}

Fuente: Elaboración propia

En cuanto al lugar y a los datos por estación hay significancia.

\section{Test de amplitud múltiple Duncan}

Promedios

$\begin{array}{ll}\text { S1 } & 8,907 \\ \text { S2 } & 8,7 \\ \text { S3 } & 7,32\end{array}$

\begin{tabular}{ll} 
I a II & 2 posiciones \\
I a III & 3 posiciones \\
II a III & 2 posiciones \\
\hline
\end{tabular}

Finalmente al no notar una amplitud y al dar como amplitud los tres lugares se procedió a
2 Posiciones 3 Posiciones

\begin{tabular}{l|ccc}
\multirow{2}{*}{$\mathrm{Rx}$} & $5 \%$ & 2,9 & 3,04 \\
& $1 \%$ & 3,91 & 4,08 \\
\multirow{2}{*}{$\mathrm{Sx} R \mathrm{Rx}$} & $5 \%$ & 0,35 & 0,37 \\
& $1 \%$ & 0,47 & 0,49 \\
\hline
\end{tabular}

\begin{tabular}{lllll} 
I a II & 2 & $-0,24$ & $<0,35$ & NOSIG \\
I a III & 3 & $-1,59$ & $<0,37$ & NOSIG \\
II a III & 2 & 1,35 & $>0,35$ & SIG \\
\hline
\end{tabular}

escoger el valor con mayor promedio que es el de Matarani con $8,91 \mathrm{mg} / \mathrm{Kg}$

Tabla 7. Análisis de varianza para el otoño

\begin{tabular}{|c|c|c|c|c|c|c|}
\hline \multirow{2}{*}{$\begin{array}{l}\text { Fuente de } \\
\text { variación }\end{array}$} & \multirow[b]{2}{*}{ GI } & \multirow[b]{2}{*}{ SC } & \multirow[b]{2}{*}{$\mathrm{CM}$} & \multicolumn{3}{|c|}{ Cálculo de F } \\
\hline & & & & F calculado & F tabulado & F tabulado \\
\hline & & & & & $5 \%$ & $1 \%$ \\
\hline Lugar & 2 & 24,35 & 12,1769 & 34,82 & 3,33 & 5.42 \\
\hline Análisis & 14 & 6,30 & 0,4502 & 1,29 & 2,05 & 2.75 \\
\hline Error & 28 & 9,79 & 0,349746032 & & & \\
\hline Total & 44 & 40,45 & 0,9193 & & & \\
\hline
\end{tabular}

Fuente: Elaboración propia 
En cuanto al lugar y al análisis de los datos por estación hay significancia

\section{Test de amplitud múltiple Duncan}

promedios

$\begin{array}{ll}\text { S1 } & 8,593333333 \\ \text { S2 } & 8,4 \\ \text { S3 } & 6,926666667\end{array}$

\section{I a II 2 posiciones}

I a III 3 posiciones

II a III 2 posiciones

\begin{tabular}{l|ccc} 
& & 2 Posiciones & 3 Posiciones \\
\hline \multirow{2}{*}{$\mathrm{Rx}$} & $5 \%$ & 2,9 & 3,04 \\
& $1 \%$ & 3,91 & 4,08 \\
\multirow{3}{*}{ Sx Rx } & $5 \%$ & 0,44 & 0,46 \\
& $1 \%$ & 0,60 & 0,62 \\
\hline
\end{tabular}

$\begin{array}{llrcc}\text { I a II } & 2 & -0,24 & <0,44 & \text { NOSIG } \\ \text { I a III } & 3 & -1,67 & <0,46 & \text { NOSIG } \\ \text { II a III } & 2 & 1,43 & >0,44 & \text { SIG }\end{array}$

escoger el valor con mayor promedio que sería el de Matarani con $8,59 \mathrm{mg} / \mathrm{Kg}$

Finalmente al no notar una amplitud y al dar como amplitud los tres lugares se procederá a

Tabla 8. Análisis de varianza para el invierno

\begin{tabular}{|c|c|c|c|c|c|c|}
\hline \multirow{2}{*}{$\begin{array}{l}\text { Fuente de } \\
\text { variación }\end{array}$} & \multirow[b]{2}{*}{ GI } & \multirow[b]{2}{*}{ SC } & \multirow[b]{2}{*}{$\mathrm{CM}$} & \multicolumn{3}{|c|}{ Cálculo de F } \\
\hline & & & & F calculado & F tabulado & F tabulado \\
\hline & & & & & $5 \%$ & $1 \%$ \\
\hline Lugar & 2 & 17,61 & 8,8062 & 32,24 & 3,33 & 5,42 \\
\hline Análisis & 14 & 7,56 & 0,5402 & 1,98 & 2,05 & 2,75 \\
\hline Error & 28 & 7,65 & 0,273126984 & & & \\
\hline Total & 44 & 32,82 & 0,7460 & & & \\
\hline
\end{tabular}

Fuente: Elaboración propia

En cuanto al lugar y a los datos por estación hay significancia.

\section{Test de amplitud múltiple Duncan}

Promedios

$$
\begin{array}{ll}
\text { S1 } & 7,086666667 \\
\text { S2 } & 7,0 \\
\text { S3 } & 5,74
\end{array}
$$

\begin{tabular}{ll} 
I a II & 2 posiciones \\
\hline I a III & 3 posiciones \\
II a III & 2 posiciones
\end{tabular}

\begin{tabular}{l|ccc} 
& & 2 Posiciones & 3 Posiciones \\
\hline $\mathrm{Rx}$ & $5 \%$ & 2,9 & 3,04 \\
& $1 \%$ & 3,91 & 4,08 \\
$\mathrm{Sx} \mathrm{Rx}$ & $5 \%$ & 0,39 & 0,41 \\
& $1 \%$ & 0,53 & 0,55
\end{tabular}

$\begin{array}{llrlrc}\text { I a II } & 2 & -0,04 & <0,39 & \text { NOSIG } \\ \text { I a III } & 3 & -1,35 & <0,41 & \text { NOSIG } \\ \text { II a III } & 2 & 1,31 & =0,39 & \text { SIG }\end{array}$


Finalmente al no notar una amplitud y al dar como amplitud los tres lugares se procedió a escoger el valor con mayor promedio que fue el de Matarani con 7,09mg/Kg

\section{t-student}

Este test permite, determinar si existen diferencias significativas (varianza) en la variable analizada, se calcula con la siguiente expresión:

$$
S^{2}=\frac{\sum X_{1}^{2}-\frac{\left(\sum X_{1}\right)^{2}}{N_{1}}+\sum X_{2}^{2}-\frac{\left(\sum X_{2}\right)^{2}}{N_{2}}}{\left(N_{1}-1\right)+\left(N_{2}-1\right)}
$$

Seguidamente calcularemos el t-student con la siguiente expresión:

$$
t=\frac{X_{1}-X_{2}}{S * \sqrt{\frac{1}{N_{1}}+\frac{1}{N_{2}}}}
$$

Así Para Matarani e Ilo se tiene:

\begin{tabular}{|ccc|}
\hline Estación & \multicolumn{2}{c}{ Lugar de Muestreo } \\
& Matarani & Ilo \\
\hline Primavera & 8,15 & 8,09 \\
\hline Verano & 8,91 & 8,67 \\
\hline Otoño & 8,59 & 8,35 \\
\hline Invierno & 7,09 & 7,05 \\
\hline Sum X1 & 32,74 & 32,16 \\
\hline X1 (prom) & 8,19 & 8,04 \\
\hline Sum.X1^2 & 269,87 & 260,04 \\
\hline
\end{tabular}

Cálculo de S

$$
\begin{aligned}
& S^{2}=0,561 \\
& S=0,749
\end{aligned}
$$

Cálculo de $\mathrm{t}$

$$
\mathrm{t}=0.274
$$

Cálculo de los grados de libertad, con la expresión:

$g l=\left(N_{1}-1\right)+\left(N_{2}-1\right)=(4-1)+(4-1)=6$

\begin{tabular}{|c|c|c|}
\hline \multirow{2}{*}{ Estación } & \multicolumn{2}{|c|}{ Lugar de Muestreo } \\
\hline & Matarani & Ilo \\
\hline Primavera & 8,15 & 6,64 \\
\hline Verano & 8,91 & 7,32 \\
\hline Otoño & 8,59 & 6,93 \\
\hline Invierno & 7,09 & 5,74 \\
\hline Sum X1 & 32,74 & 26,63 \\
\hline $\mathrm{X} 1$ (prom) & 8,19 & 6,66 \\
\hline Sum.X1^2 & 269,87 & 178,64 \\
\hline
\end{tabular}

luego para 6 grados de libertad se busca en tablas (anexo) al $5 \%$ se tiene un $t$ de tabla de 2,4469

$\begin{array}{ccc}\mathrm{t} \text { calculado } & \mathrm{t} \text { tabla } & \text { significancia } \\ 0,274 & 2,4469 & \text { no significante } \\ \mathrm{t} \text { cal }<\mathrm{t} \text { tabla }\end{array}$

En cuanto a la evaluación Matarani - Vila Vila :

Seguidamente se calcula $S$

$$
\begin{aligned}
& S^{2}=0,541 \\
& S=0,735
\end{aligned}
$$

\begin{tabular}{|c|c|c|}
\hline t calculado & $\mathrm{t}$ tabla significancia & \\
\hline 2,937 & significante & $\mathrm{t} \mathrm{cal}<\mathrm{t}$ tabla \\
\hline
\end{tabular}

Cálculo de t

$$
\mathrm{t}=2.937
$$

Cálculo de los grados de libertad con la expresión: $g l=\left(N_{1}-1\right)+\left(N_{2}-1\right)=(4-1)+(4-1)=6$

luego para 6 grados de libertad y usando la tabla 1 (anexo) al $5 \%$ se tiene un te tabla de 2,4469

Entonces hay mucha significancia entre realizar el muestreo en Matarani o Vila Vila. 
Finalmente la relación Ilo - Vila Vila

\begin{tabular}{|ccc|}
\hline Estación & \multicolumn{2}{c|}{ Lugar de Muestreo } \\
& Ilo & Vila Vila \\
\hline Primavera & 8,09 & 6,64 \\
\hline Verano & 8,67 & 7,32 \\
\hline Otoño & 8,35 & 6,93 \\
\hline Invierno & 7,05 & 5,74 \\
\hline Sum X1 & 32,16 & 26,63 \\
\hline X1 (prom) & 8,04 & 6,66 \\
\hline Sum.X1^2 & 260,04 & 178,64 \\
\hline
\end{tabular}

Calculamos S

$$
\begin{aligned}
& S^{2}=0,472 \\
& S=0,687
\end{aligned}
$$

Cálculo de $\mathrm{t}$

$$
t=2,846
$$

Cálculo de los grados de libertad con la expresión:

$g l=\left(N_{1}-1\right)+\left(N_{2}-1\right)=(4-1)+(4-1)=6$

\begin{tabular}{|c|c|c|c|}
\hline t calculado & t tabla & significancia & \\
\hline 2,846 & 2,4469 & significante & $\mathrm{t}$ cal $<\mathrm{t}$ tabla \\
\hline
\end{tabular}

luego para 6 grados de libertad y usando la tabla 1 (anexo) al $5 \%$, se tiene un t de tabla de 2,4469

Entonces hay mucha significancia en realizar el muestreo en Ilo o en Vila Vila.

Los resultados promedio finales fueron:

Tabla 9. Concentraciones de metil mercurio por zona de influencia

\begin{tabular}{lccc}
\multicolumn{1}{r}{ Zona / Estación } & Matarani & Ilo & Vila Vila \\
\hline Primavera $(\mathrm{mg} / \mathrm{Kg})$ & 8,15 & 8,09 & 6,64 \\
\hline Verano $(\mathrm{mg} / \mathrm{Kg})$ & 8,91 & 8,67 & 7,32 \\
\hline Otoño $(\mathrm{mg} / \mathrm{Kg})$ & 8,59 & 8,35 & 6,93 \\
\hline Invierno $(\mathrm{mg} / \mathrm{Kg})$ & 7,09 & 7,05 & 5,74
\end{tabular}

Fuente: Elaboración propia

\section{CONCLUSIONES}

- Se observa que en el tratamiento estadístico en la primera parte del análisis de varianza no indica preferencia por alguna zona debido a la significancia y en el análisis Duncan, por la amplitud de la misma que abarca las tres zonas de muestreo. Indica que la zona de mayor promedio en mercurio es Matarani
- Las tres localidades presentan concentraciones de metil mercurio superiores a los valores permitidos por la OMS ( $5 \mathrm{mg} / \mathrm{kg}$ ), pero los valores más altos encontrados corresponden al sector de Matarani.

- Se observa también que los valores más altos comprendena las estaciones de verano y otoño, correspondientes a estaciones aluviales en los andes. 


\section{BIBLIOGRAFÍA}

1 ATSDR (Agency for Toxic Substances and Disease Registry). (1993). Toxicological Profilefor Mercury (Update). Atlanta, GA: US Department of Health and Human Services.

2. Barrado A. (1995). El Pescado y las Industrias derivadas de la Pesca. Editorial Zaragoza

3. Cortinas de Nava, C; Ruiz Ruiz, F; Cristán Frías, A.(1996).Lo que usted debesabersobreelmercurio y su situación en América de Norte, Serie Mercurio No.1 . México. D.F.: INE/SEMARNAP.

4. Mason, R.P., Morel F.M.M., y Hemond H.F. (1995). The role of microorganisms in elemental mercury formation in natural waters. (El rol de los microorganismos en la formación del mercurio elemental en aguas naturales). Water, Air and Soil Pollution

5. Mason, R.P., Laporte J.M., y Andres S. (2000). Factors controlling the bioaccumulation of mercury, methylmercury, arsenic, selenium, and cadmium by freshwater invertebrates and fish. (Factores que Controlan la bioacumulación de mercurio, metilmercurio, arsénico, selenio y cadmio por invertebrados y peces de agua dulce). Archives of Environmental Contamination and Toxicology

6. Mckim, J. M., Olson G. F., Holcombe G. W., y Hunt E. P. (1976). Long-term effects of methylmercuric chloride on three generations of brook trout (Salvelinus fonyinalis): toxicity, accumulation, distribution and elimination. (Efectos a largo plazo del cloruro metilmercúrico en tres generaciones de truch a de arroyo (Salvelinus fonyinalis), Journal of the Fisheries Research Board of Canada

7. Slotton, D.G., Reuter J.E., y Goldman C.R. (1995). Mercury uptake patterns of biota in a seasonally anoxic Northern California reservoir. (Patrones de ingesta de mercurio de la biota en un reservorio estacionalmente anóxico de California del Norte). Water, Air and Soil Pollution 80.

Correspondencia: Anyelina Paz e-mail: anyelinapaz@yahoo.es 